\title{
Charged Forms of Diacetone Diperoxide - DFT Treatment
}

\section{Lemi Türker}

Department of Chemistry, Middle East Technical University, Üniversiteler, Eskişehir Yolu No: 1, 06800 Çankaya/Ankara, Turkey; e-mail: 1turker@gmail.com; lturker@metu.edu.tr

\begin{abstract}
Diacetone diperoxide (DADP) is one of the sensitive and powerful organic peroxide explosives like its trimeric analogue TATP. Presently, some ionic forms of it have been investigated within the limitations of density functional theory at the level of UB3LYP/6$311++\mathrm{G}(\mathrm{d}, \mathrm{p})$. Various properties (including structural, electronic, spectral and quantum chemical) have been obtained and discussed. The studied mono and dianions having different multiplicity states have been found to be stable but monocation form decomposed.
\end{abstract}

\section{Introduction}

Triacetone triperoxide (TATP), diacetone diperoxide (DADP) and hexamethylene triperoxide diamine (HMTD) are sensitive and powerful organic peroxide explosives and have been used from time to time by terrorists in the past. They are quite attractive to terrorists because of the syntheses are straightforward, which require only a few easily obtainable ingredients. Generally, organic peroxides are a relatively unexplored class of substances. The most prominent example is acetone peroxide which was synthesized for the first time by the German chemist Richard Wolffenstein in 1895 [1]. Some detailed investigations concerning the composition of the product were performed later and it was found that the main fraction is a trimeric molecule (triacetone tri peroxide, TATP, 3,3,6,6,9,9-hexamethyl-1,2,4,5,7,8-hexoxonane). The trimer is accompanied by a dimer, diacetone diperoxide (DADP) [2]. The ratio of the two possible products are formed depends on the reaction conditions (mostly the concentration of the acid used) employed during the reaction. Generally, concentrated and strong acids favor the formation of

Received: October 24, 2021; Accepted: November 25, 2021

Keywords and phrases: DADP; diacetone diperoxide; peroxide explosives; explosives; impact sensitivity.

Copyright (C) 2022 Lemi Türker. This is an open access article distributed under the Creative Commons Attribution License, which permits unrestricted use, distribution, and reproduction in any medium, provided the original work is properly cited. 
DADP whereas lower acid concentrations or acid free conditions lead to the formation of TATP. Under the catalysis of tin (IV), a tetrameric form can be isolated [3].

Bali et al., have noted that research to counter the threat of organic peroxides such as triacetone triperoxide (TATP) is at times hampered by their inherent extreme sensitiveness and volatility [4].

Oxley et al., have discussed methods to degrade peroxide explosives, DADP, TATP, and HMTD, chemically, at room temperature. A number of mixtures containing metals (e.g., zinc, copper) and metal salts (e.g., zinc sulfate, copper chloride) were found to be effective, so that some capable of destroying TATP solutions in a few hours. Strong acids proved useful against solid peroxide materials [5].

Of the two acetone peroxides, diacetone diperoxide (DADP) is the thermodynamically-favored one, as opposed to the more commonly formed and kinetically-favored triacetone triperoxide (TATP). As a 6-membered ring, DADP possessed significantly more inherent bond strain than its larger, 9-membered counterpart. Research by Matyáš et al. has shown friction sensitivity differences between DADP, HMTD and TATP [6]. The other research has indicated that conversion of TATP to DADP can occur spontaneously if conditions are favorable [7]. On the other hand, vapor pressures of both acetone peroxides are significant to be detected by the human olfactory sense [8]. It is still debated on which pathway(s) acetone and hydrogen peroxide follow to form TATP and whether DADP forms directly, or if TATP forms first followed by rearrangement to DADP in solution [9]. It has been reported that the synthesis of TATP occurs slowly over time when hydrogen peroxide (HP) and acetone are combined for a sufficiently long period, whereas the addition of an acid catalyst significantly accelerates the process $[1,10]$. However, with addition of low to moderate levels of acid catalyst, a mixture of TATP and DADP will form, but at high levels of added acid DADP forms as the sole product. Although these compounds are regarded more as an illicit threats, recent studies have indicated interesting behavior of DADP when co-crystallized with other explosive materials [11]. Characterization of diacetone diperoxide has been achieved by Bowden et al., [12]. On the other hand, using density functional theory calculations Kahnooji et al., investigated relationships between the structures and performance of a series of highly energetic diacetone diperoxides [13].

\section{Method of Calculations}

All the structures were subjected to MM2 initial geometry optimizations leading to 
energy minima followed by semi-empirical PM3 self-consistent fields molecular orbital (SCF MO) method [14,15] at the restricted level [16]. Then, the structure optimizations have been achieved within the framework of Hartree-Fock (HF) and finally by using density functional theory (DFT) at the level of UB3LYP/6-311++G(d,p) [17,18]. The exchange term of B3LYP consists of hybrid Hartree-Fock and local spin density (LSD) exchange functions with Becke's gradient correlation to LSD exchange [19]. The correlation term of B3LYP consists of the Vosko, Wilk, Nusair (VWN3) local correlation functional [20] and Lee, Yang, Parr (LYP) correlation correction functional [21]. The normal mode analysis for each structure yielded no imaginary frequencies for the $3 \mathrm{~N}-6$ vibrational degrees of freedom. Note that $N$ is the number of atoms in the system and the present normal mode analysis indicates that the structure of each molecule corresponds to at least a local minimum on the potential energy surface. Furthermore, all the bond lengths were thoroughly searched in order to find out whether any bond cleavage occurred or not during the geometry optimization process. All these computations were performed by using SPARTAN 06 [22].

\section{Results and Discussion}

Compounds which contain bonds with relatively low bond dissociation energies decompose into radicals by certain means such as heat [23]. The commonest is the peroxidic bond, O-O, in dialkyl peroxides, $\mathrm{RO}-\mathrm{OR}$ and diacyl peroxides, $\mathrm{RC}(=\mathrm{O}) \mathrm{O}-$ $\mathrm{OC}(=\mathrm{O}) \mathrm{R}[23,24]$. Some of them such as DADP and TATP possess explosive properties.

Figure 1 shows the optimized structures and the direction of the calculated dipole moment vectors of the species presently considered. In the figure abbreviations, ma, da,
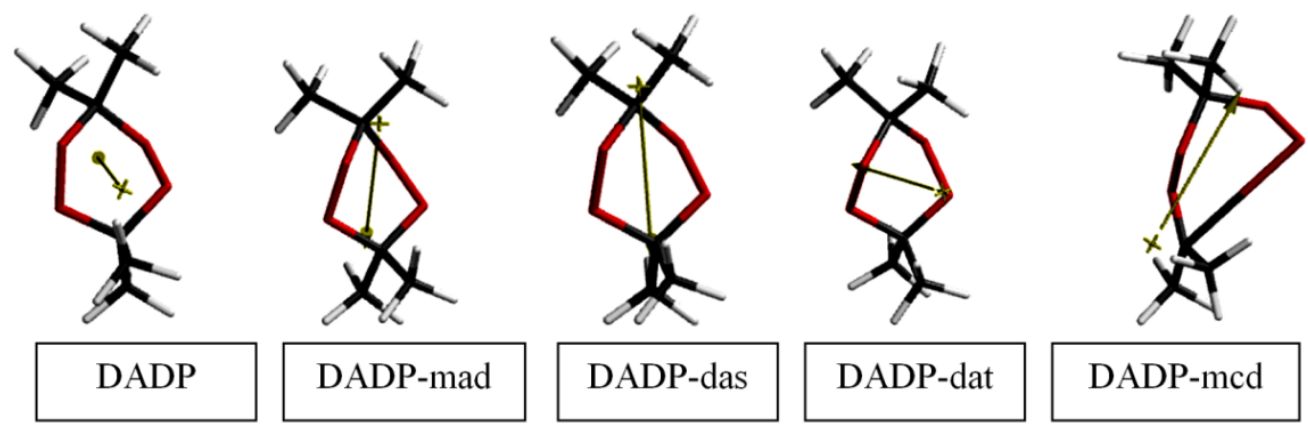

Figure 1. Optimized structures of the species considered.

and me stand for monoanion, dianion and monocation, respectively. They are followed by letters $\mathrm{d}, \mathrm{s}$ and $\mathrm{t}$ which indicate either singlet, doublet or triplet case. Note that in 
almost all the cases the dipole moment originates from somewhere nearby one of the ring carbon atoms to somewhere nearby the other ring carbon atom. The dianion in the triplet case is somewhat different from the others in which the dipole moment vector lies from one of the peroxide linkage to the other. Note that monocation doublet (DADP-mcd) has been characterized with a ruptured $\mathrm{C}-\mathrm{O}$ bond.

Figure 2 shows the bond lengths/ distances of the species presently considered. As
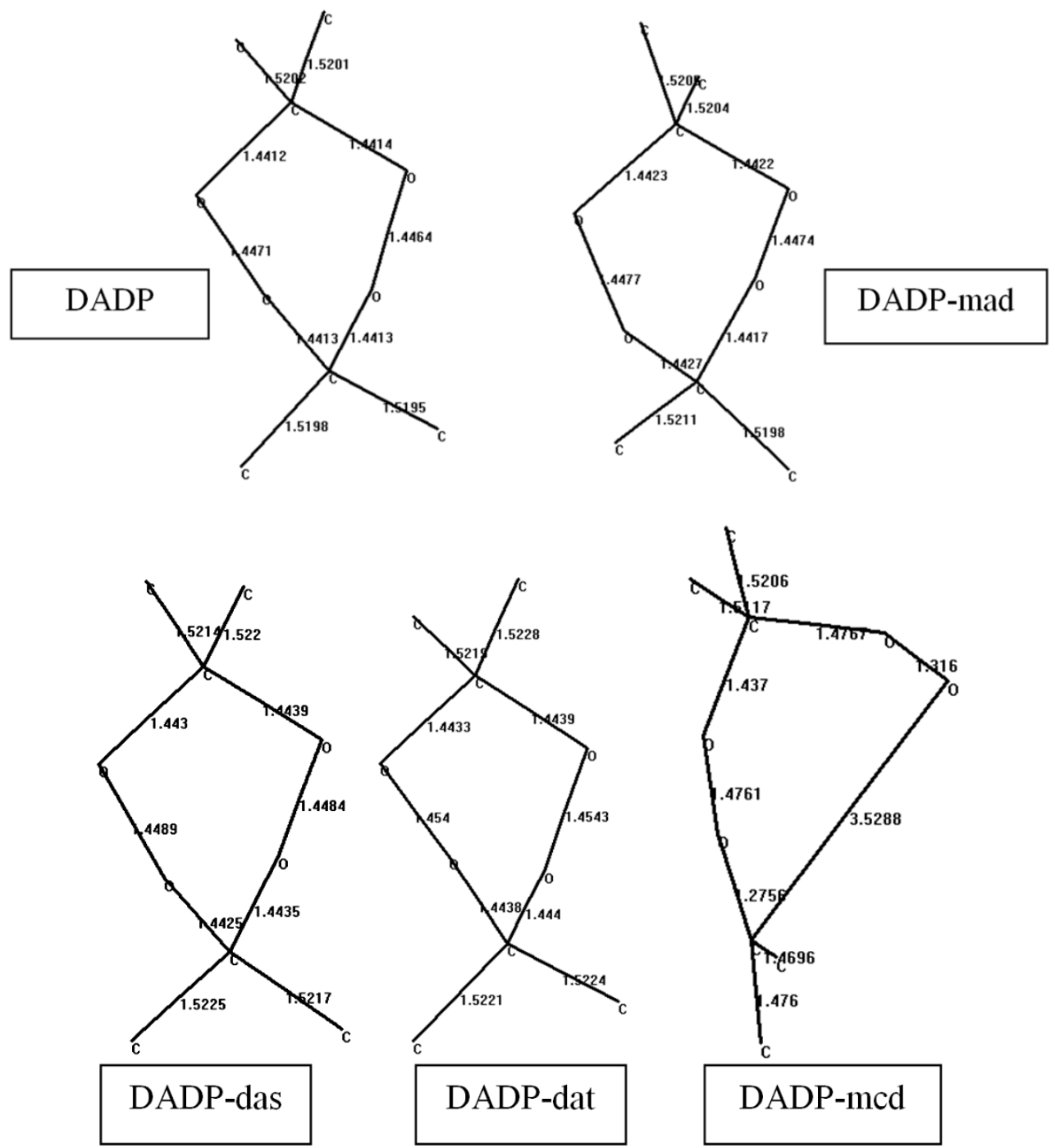

Figure 2. Calculated bond lengths/distances of the species considered (Hydrogens omitted). 
seen in the figure peroxide and $\mathrm{C}-\mathrm{O}$ bond lengths in the structures with the exception of decomposed cation form are almost constant and comparable with respective bond lengths in DADP. However, conformations of them are quite different. It has to be dictated by partitioning of the excess charge among the atoms.

The optimized structures have methyl groups which are twos by twos are on 1,4positions. Each pair of methyl groups is in different planes which are oblique or perpendicular to each other. The cleavage of $\mathrm{C}-\mathrm{O}$ bond in monocation case is somewhat unexpected at first sight because the peroxide linkage in organic peroxides is expected to be more susceptible to bond cleavage. However, this statement is true for thermal decomposition of the peroxides whereas presently charged forms of DADP are of interest.

Table 1 lists some of the properties of the species considered. For all the structures except the cation the properties considered are quite comparable. The cation on the other hand, possesses comparatively a large dipole moment value.

Table 1. Some properties of the species considered.

\begin{tabular}{lcccc}
\hline \multicolumn{1}{c}{ Structure } & Area $\left(\AA^{2}\right)$ & Volume $\left(\AA^{3}\right)$ & $\begin{array}{c}\text { Dipole moment } \\
(\text { Debye })\end{array}$ & Ovality \\
\hline DADP & 177.35 & 149.96 & 0.01 & 1.30 \\
DADP-mad & 177.58 & 150.10 & 0.03 & 1.30 \\
DADP-das & 177.79 & 150.21 & 0.09 & 1.30 \\
DADP-dat & 177.97 & 150.29 & 0.07 & 1.30 \\
DADP-mcd & 189.32 & 155.49 & 4.88 & 1.35 \\
\hline
\end{tabular}

Figures 3 and 4 display the electrostatic potential charges (ESP) and electrostatic potential maps of the species of present concern, respectively. Note that the ESP charges are obtained by the program based on a numerical method that generates charges that reproduce the electrostatic potential field from the entire wavefunction [22]. As seen in the figure, the distribution of charges in DADP is quite symmetrical. This is almost valid for the anions of the present concern. The charges on the oxygen atoms are all negative and all the methyl carbons possess certain negative charges too. The positive charges reside on the ring carbon atoms even in some cases (anions) greater than unity. 

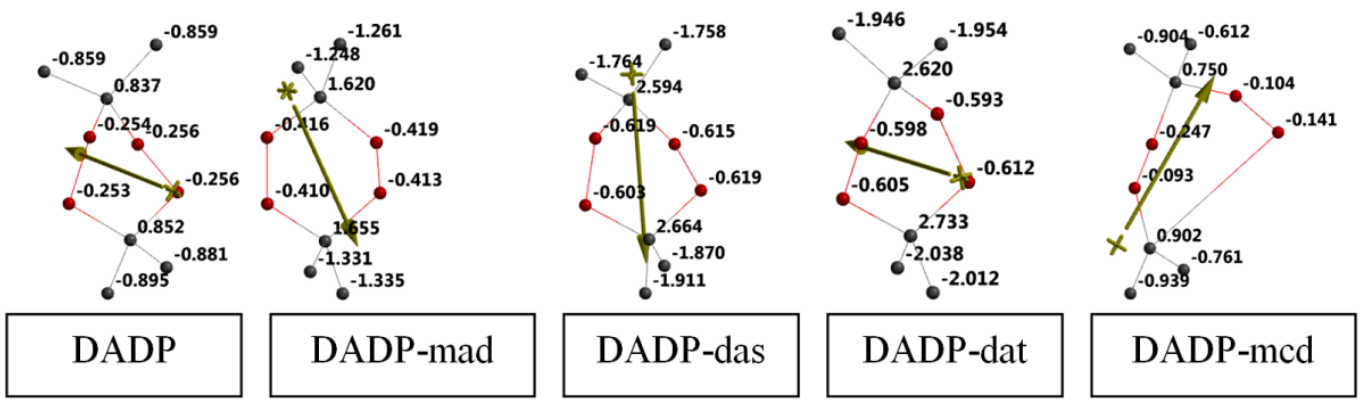

Figure 3. Electrostatic potential charges of the species considered (Hydrogens omitted).
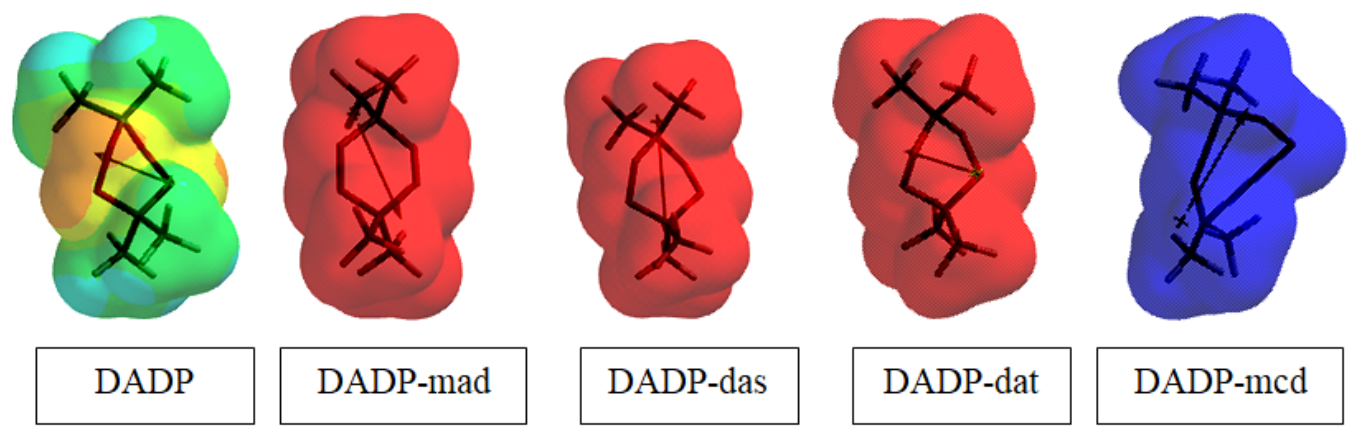

Figure 4. Electrostatic potential maps of the species considered.

Table 2 list some energies of the species considered where $\mathrm{E}, \mathrm{ZPE}$ and $\mathrm{E}_{\mathrm{C}}$ stand for the total electronic energy, zero point vibrational energy and the corrected total electronic energy, respectively. Note that of the dianions considered, the triplet is more stable than the singlet.

Table 2. Some energies of the species considered.

\begin{tabular}{lccc}
\hline \multicolumn{1}{c}{ Structure } & $\mathbf{E}$ & $\mathbf{Z P E}$ & $\mathbf{E}_{\mathbf{C}}$ \\
\hline DADP & -1409225.31 & 473.98 & -1408751.33 \\
DADP-mad & -1409172.75 & 470.33 & -1408702.42 \\
DADP-das & -1408900.36 & 468.83 & -1408431.53 \\
DADP-dat & -1408915.97 & 468.35 & -1408447.62 \\
DADP-mcd & -1408404.87 & 466.15 & -1407938.72 \\
\hline
\end{tabular}

Energies in $\mathrm{kJ} / \mathrm{mol}$. 
Figure 5 shows some of the molecular orbital energy levels of the species considered.
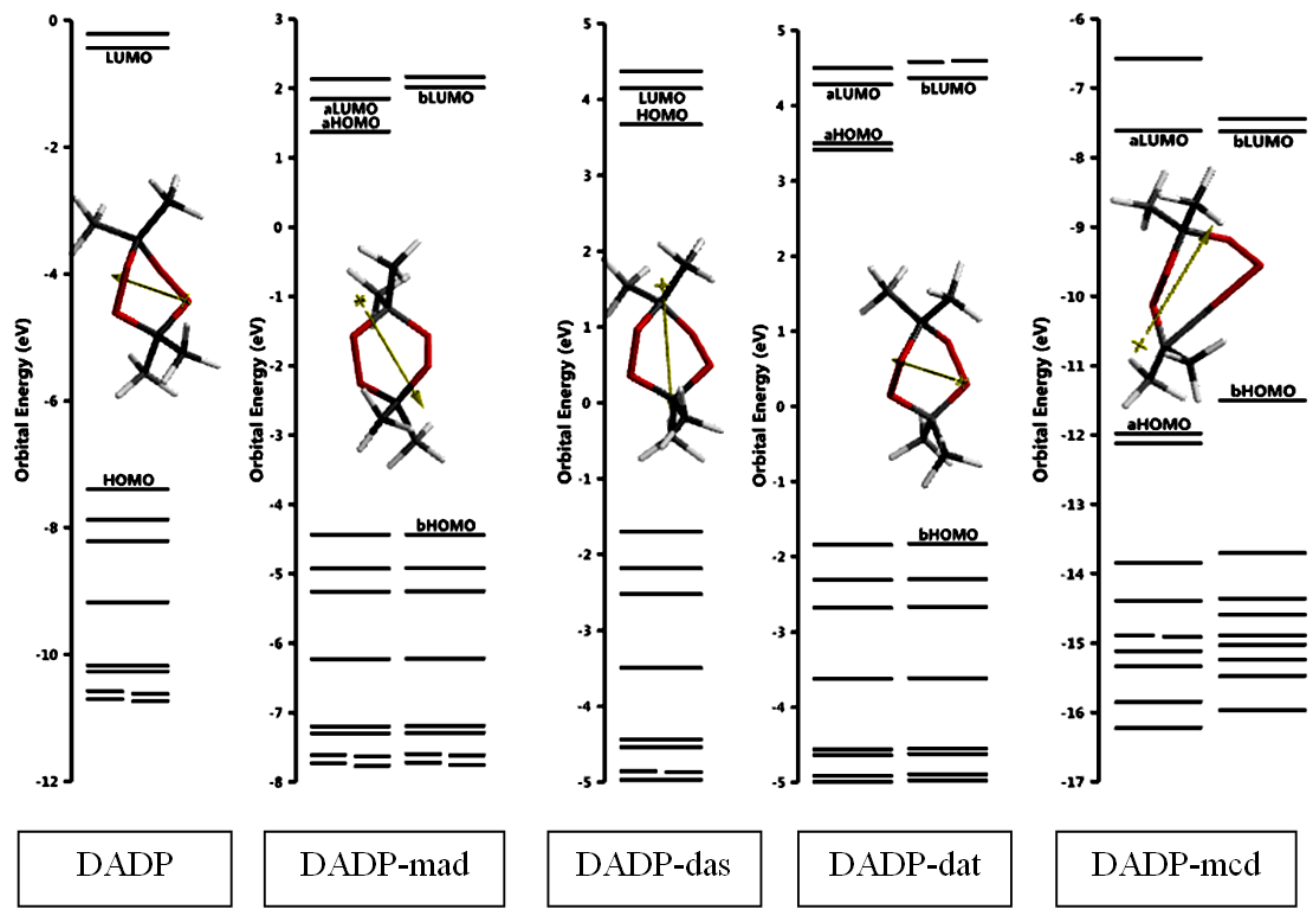

Figure 5. Some molecular orbital energy levels of the species considered.

As seen in the figure the open-shell systems possess $\alpha$ - and $\beta$-type molecular orbitals which are indicated in the figure by as a- and b-types. Table 3 shows the HOMO, LUMO energies and the interfrontier molecular orbital energy gap $(\Delta \varepsilon)$ values of the present systems. As seen in the table, charging DADP molecule negatively raises up the HOMO and LUMO energies. The energy order is DADP-med $<$ DADP $<$ DADP-mad $<$ DADPdat $<$ DADP-das and DADP-mcd $<$ DADP $<$ DADP-mad $<$ DADP-das $<$ DADP-dat, respectively for the HOMO and LUMO energies. As for the $\Delta \varepsilon$ values the order is DADP-mad $<$ DADP-das $<$ DADP-dat $<$ DADP-mcd $<$ DADP. However, note that DADP-mcd is a decomposed one in the set. Considering the rest of the systems only, the calculations indicate that transforming DADP into anionic forms narrows the interfrontier energy gap (frontier molecular orbital energy gap, FMO gap) considerably. Since impact sensitivity has been correlated with FMO gap reversely [25,26], the order of sensitivity to impact stimulus should follow the order of DADP-mad > DADP-das > DADP-dat $>$ DADP. 
Table 3. The HOMO, LUMO energies and $\Delta \varepsilon$ values of the species considered.

\section{Structures}

\begin{tabular}{lccccc}
\hline & DADP & DADP-mad & DADP-das & DADP-dat & DADP-mcd \\
\hline LUMO & -41.94 & 178.18 & 400.29 & 413.49 & -733.84 \\
HOMO & -713.53 & 132.35 & 354.45 & 337.39 & -1155.66 \\
$\boldsymbol{\Delta} \boldsymbol{\varepsilon}$ & 671.59 & 45.83 & 45.84 & 76.1 & 421.82 \\
\hline
\end{tabular}

Energies in $\mathrm{kJ} / \mathrm{mol}$.

Figures 6 and 7 show the HOMO and LUMO patterns of the species, respectively.
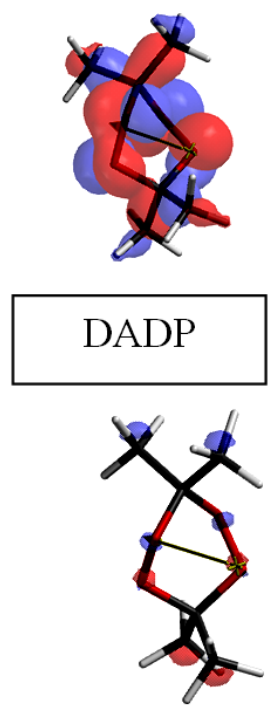

DADP-dat $\alpha$
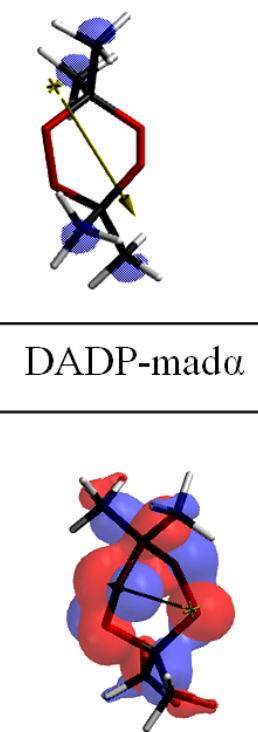

DADP-dat $\beta$

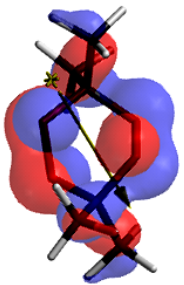

\section{DADP-mad $\beta$}

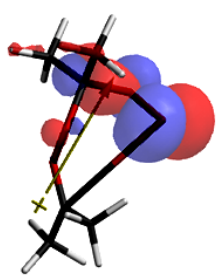

DADP- $\operatorname{mcd} \alpha$

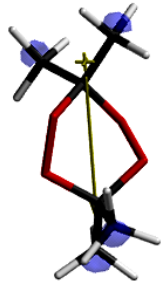

DADP-das

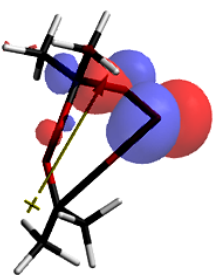

DADP- $\operatorname{mcd} \beta$

Figure 6. The HOMO patterns of the species considered. 

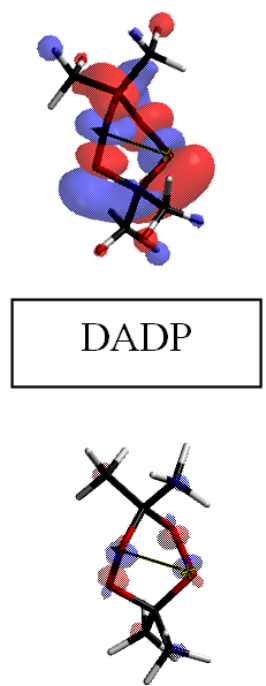

DADP-dat $\alpha$
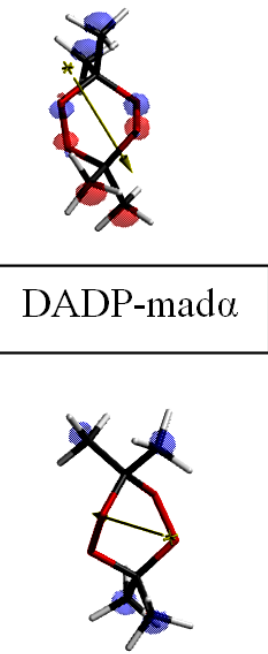

DADP-dat $\beta$
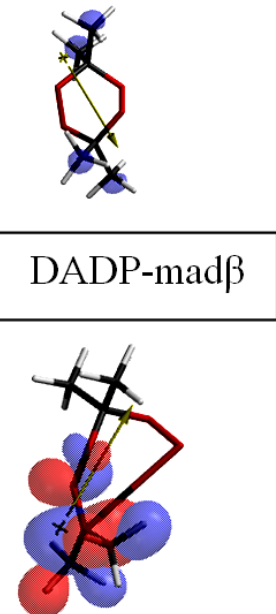

DADP- $\operatorname{mcd} \alpha$

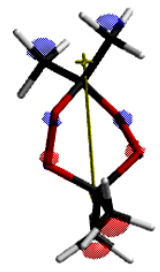

DADP-das

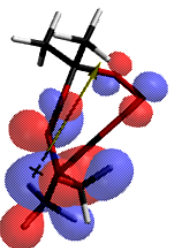

Figure 7. The LUMO patterns of the species considered.

Figure 8 shows the calculated UV-VIS spectra of DADP and the decomposed mono
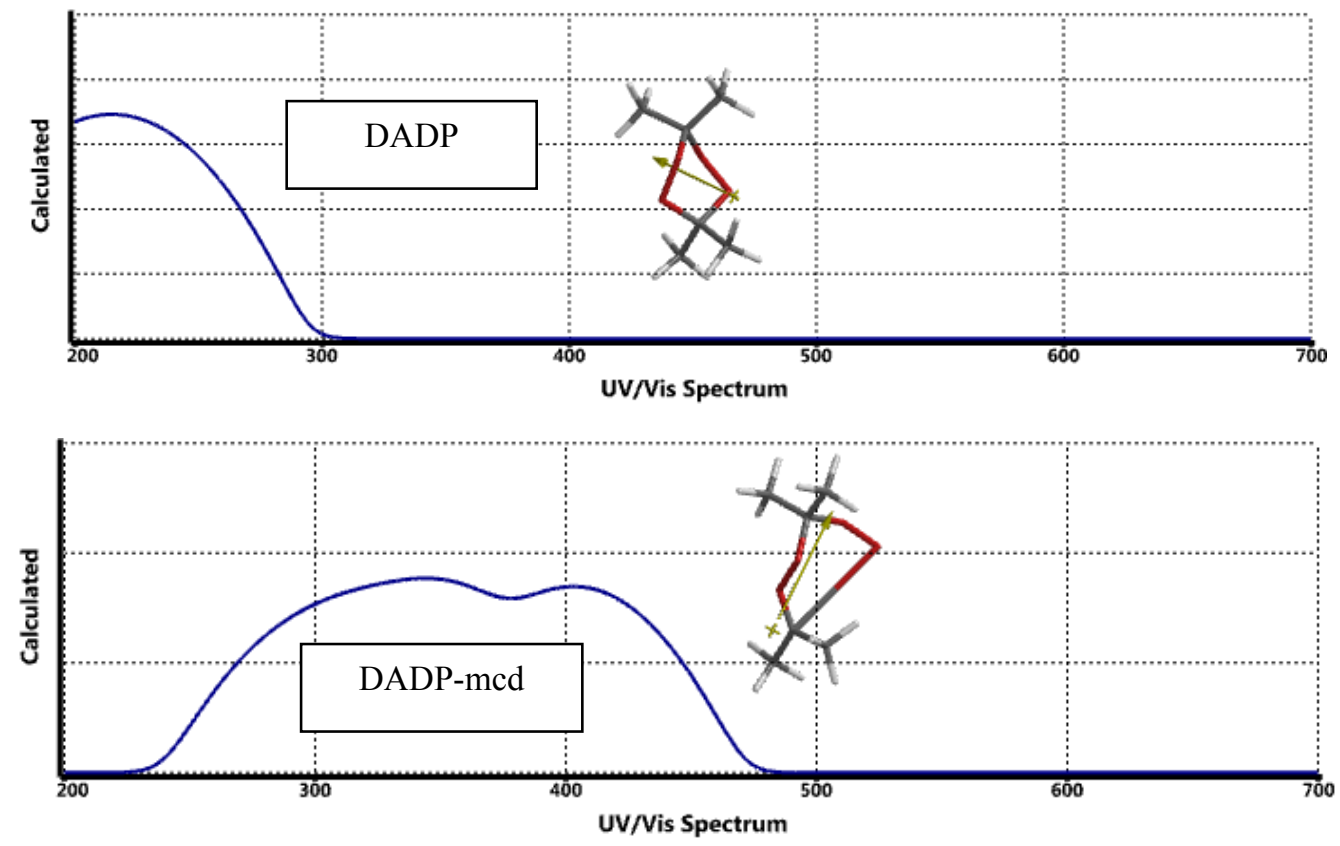

Figure 8. Calculated UV-VIS spectra of DADP and DADP-mcd. 
cation of it. The calculated spectra of the anions do not exhibit any absorption in the UVVIS range although the interfrontier molecular orbital energy gaps for them are narrower then the corresponding value for DADP. The reason could be very small transition moment existing for the HOMO-LUMO transition of them [27-29].

Figure 9 shows the spin densities of the open-shell structures considered. As seen in the figure in the case of anion the spin density is concentrated around methyl carbons. Whereas in the decomposed system (monocation form) the relatively highest spin density is on the oxygen atom of the ruptured $\mathrm{C}-\mathrm{O}$ bond.
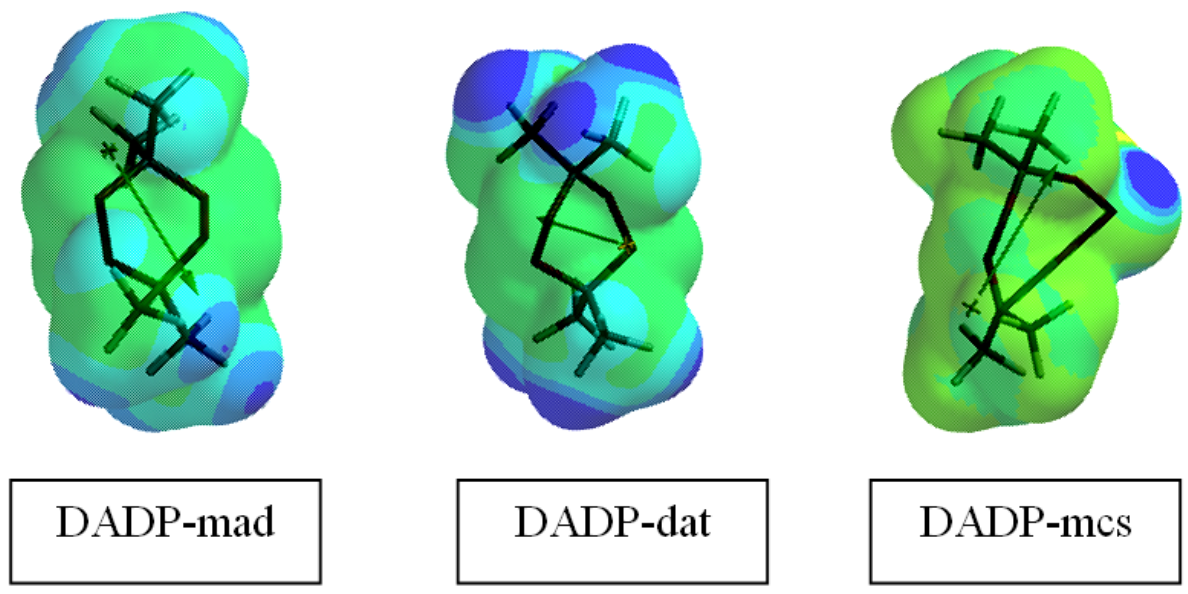

Figure 9. Spin densities of the open-shell structures considered.

Figure 10 shows the local ionization potential maps of the systems considered. In the local ionization potential map conventionally red regions on the density surface indicate
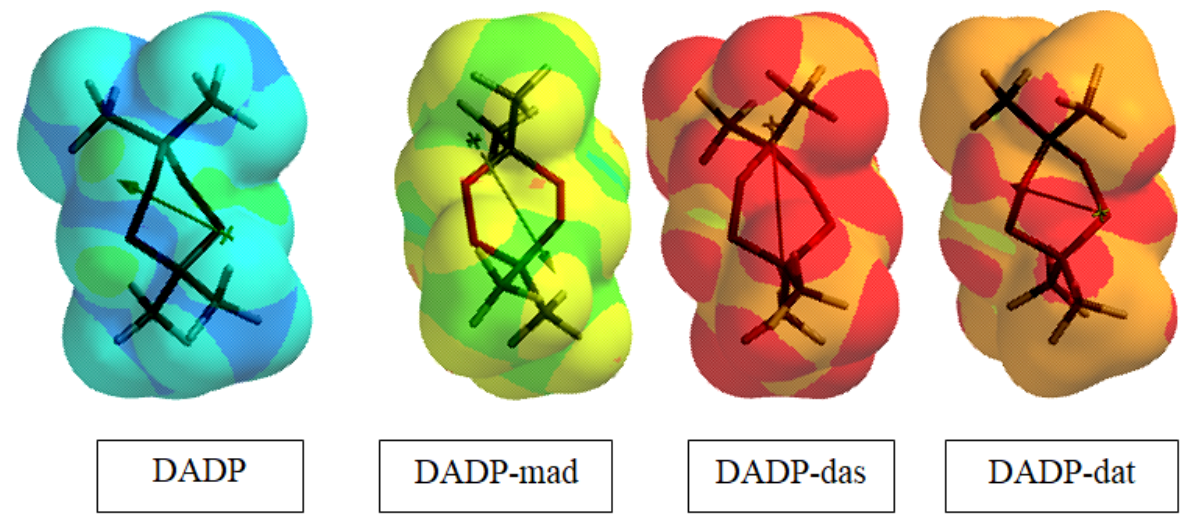

Figure 10. The local ionization potential maps of DADP and some of its anions. 
areas from which electron removal is relatively easy, meaning that they are subject to electrophilic attack. On the other hand, regions having blue color represent areas where ionization is relatively difficult. Note that the property is multiplicity dependent even in the species having the same charge.

\section{Conclusion}

The present DFT study, within the constraints of the theory and the basis set employed has revealed that DADP molecule can be in mono and dianion forms keeping its integrity. Whereas, it decomposes even in the mono cation form by the rupture of not the $\mathrm{O}-\mathrm{O}$ bond but $\mathrm{C}-\mathrm{O}$ linkage. On the other hand, the calculations predict that all the anionic forms are more susceptible to impact stimulus than DADP is. The mono anion is the most sensitive of all. Thus, DADP should posses high danger of static charging and impact.

\section{References}

[1] Wolffenstein, R. (1895). Ueber die Einwirkung von Wasserstoffsuperoxyd auf Aceton und Mesityloxyd. Ber. Dtsch. Chem. Ges., 28(2), 2265-2269. https://doi.org/10.1002/cber.189502802208

[2] Matyas, R., \& Pachman, J. (2010). Study of TATP: Influence of reaction conditions on product composition. Propell. Explos. Pyrotech., 35, 31-37.

https://doi.org/10.1002/prep.200800044

[3] Jiang, H., Chu, G., Gong, H., \& Qiao, Q. (1999). Tin chloride catalyzed oxidation of acetone with hydrogen peroxide to tetrameric acetone peroxide. J. Chem. Res., 28(4), 288-289. https://doi.org/10.1039/a809955c

[4] Bali, M.S., Wallace, L., Day, A.I., \& Armitt, D. (2014). Cyclic pentanone peroxide: Sensitiveness and suitability as a model for triacetone triperoxide. Journal of Forensic Sciences, 59, 936-942. https://doi.org/10.1111/1556-4029.12439

[5] Oxley, J., Smith, J.L., Huang, J., \& Luo, W. (2009). Destruction of peroxide explosives. Journal of Forensic Sciences, 54(5), 1029-1033. https://doi.org/10.1111/j.1556-4029.2009.01130.x

[6] Matyáš, R., Selesovsky, J., \& Musil, T. (2012). Sensitivity to friction for primary explosives. J. Hazard. Mater., 213-214, 236-241. https://doi.org/10.1016/j.jhazmat.2012.01.085

[7] Matyáš, R., Pachman, J., \& Ang, H.-G. (2009). Study of TATP: Spontaneous 
transformation of TATP to DADP. Propell. Explos. Pyrot., 34(6), 484-488. https://doi.org/10.1002/prep.200800043

[8] Oxley, C., Smith, J.L., Luo, W., \& Brady, J. (2009). Determining the vapor pressures of diacetone diperoxide (DADP) and hexamethylene triperoxide diamine (HMTD). Propell. Explos. Pyrot., 34(6), 539-543. https://doi.org/10.1002/prep.200800073

[9] Oxley, J.C., Smith, J.L., Steinkamp, L., \& Zhang, G. (2013). Factors influencing triacetone triperoxide (TATP) and diacetone diperoxide (DADP) formation: Part 2. Propell. Explos. Pyrot., 38(6), 841-851. https://doi.org/10.1002/prep.201200215

[10] Oxley, J.C., Smith, J.L., Bowden, P.R., \& Rettinger, R.C. (2013). Factors influencing triacetone triperoxide (TATP) and diacetone diperoxide (DADP) formation: Part I. Propell. Explos. Pyrot., 38(2), 244-254. https://doi.org/10.1002/prep.201200116

[11] Landenberger, K.B., Bolton, O., \& Matzger, A.J. (2015). Energetic-energetic cocrystals of diacetone diperoxide (DADP): Dramatic and divergent sensitivity modifications via cocrystallization. J. Am. Chem. Soc., 137, 5074-5079.

https://doi.org/10.1021/jacs.5b00661

[12] Bowden, P.R., Tappan, B.C., Manner, V.W., Preston, D.N., \&. Scott, B.L. (2017). Characterization of diacetone diperoxide (DADP). AIP Conference Proceedings, 1793, 040010. https://doi.org/10.1063/1.4971504

[13] Kahnooji, M., Pandas, H.M., Mirzaei, M., \& Peyghan, A.A. (2015). Explosive properties of nanosized diacetone diperoxide and its nitro derivatives: a DFT study. Monatsh Chem., 146(9), 1401-1408. https://doi.org/10.1007/s00706-015-1419-6

[14] Stewart, J.J.P. (1989). Optimization of parameters for semi empirical methods I. $J$. Comput. Chem., 10, 209-220. https://doi.org/10.1002/jcc.540100208

[15] Stewart, J.J.P. (1989). Optimization of parameters for semi empirical methods II. $J$. Comput. Chem., 10, 221-264. https://doi.org/10.1002/jcc.540100209

[16] Leach, A.R. (1997). Molecular modeling. Essex: Longman.

[17] Kohn, W., \& Sham, L.J. (1965). Self-consistent equations including exchange and correlation effects. Phys. Rev., 140, 1133-1138.

https://doi.org/10.1103/PhysRev.140.A1133

[18] Parr, R.G., \& Yang, W. (1989). Density functional theory of atoms and molecules. London: Oxford University Press.

[19] Becke, A.D. (1988). Density-functional exchange-energy approximation with correct asymptotic behavior. Phys. Rev. A, 38, 3098-3100.

https://doi.org/10.1103/PhysRevA.38.3098 
[20] Vosko, S.H., Vilk, L., \& Nusair, M. (1980). Accurate spin-dependent electron liquid correlation energies for local spin density calculations: a critical analysis. Can. J. Phys., 58, 1200-1211. https://doi.org/10.1139/p80-159

[21] Lee, C., Yang, W., \& Parr, R.G. (1988). Development of the Colle-Salvetti correlation energy formula into a functional of the electron density. Phys. Rev. B, 37, 785-789. https://doi.org/10.1103/PhysRevB.37.785

[22] SPARTAN 06 (2006). Wavefunction Inc. Irvine CA, USA.

[23] Norman, R.O.C. (1970). Principles of organic synthesis. London: Methuen.

[24] Fuson, R.C. (1962). Reactions of organic compounds. New York: Wiley.

[25] Anbu, V., Vijayalakshmi, K.A., Karunathan, R., Stephen, A.D., \& Nidhin, P.V. (2019). Explosives properties of high energetic trinitrophenyl nitramide molecules: A DFT and AIM analysis. Arabian Journal of Chemistry, 12(5), 621-632.

https://doi.org/10.1016/j.arabjc.2016.09.023 